\title{
L'ANALYYSE DU DISCOURS À L'AUNE D'UN QUESTIONNEMENT ÉTHIQUE
}

RÉSUMÉ: Le Dictionnaire de l'analyse du discours, publié sous la direction de Charaudeau et Maingueneau 2002, ne contient pas d'entrée "Éthique" en dépit du fait qu'il définit, par exemple, des concepts comme "sujet", "identité”, "ethos", "argumentation”, "valeur", "positionnement", "prise en charge" de la "vérité" référentielle ou de celle des dires des autres énonciateurs intégrés dans la trame polyphonique du discours. Tout se passe donc comme si l'AD avait pressenti et instauré les conditions de possibilité d'un questionnement éthique sans toutefois souhaiter que ce questionnement soit intégré dans son champ, aux côtés de la "prise en charge" de la "vérité", de la "prise en charge" des jugements de valeur par le "locuteur" ou les autres "sujets du discours". Cet article a pour fin de problématiser ces paradoxes et de tenter de démontrer qu'ils sont liés à l'une des différences majeures qui séparent l'AD du champ de l'argumentation: le rapport au "Vrai", aux valeurs et aux interactions langagières conflictuelles.

MOTS-CLÉS: Analyse du discours; argumentation; éthique; rationalité; l'Autre dans l'AD et dans la mise en mots argumentative; éthique du chercheur en sciences du langage.

\section{AVANT-PROPOS}

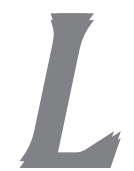

e concept d' "éthique" ne figure pas parmi les notions qui ont droit à une entrée à part entière dans le Dictionnaire d'analyse du discours, publié en 2002 sous la direction de Patrick Charaudeau et Dominique Maingueneau. On y trouve certes des références aux questions suivantes: la mise en scène et la prise en charge de divers types de vérité (référentiel,

\footnotetext{
Université Bar-Ilan, Israel.
} 
modalisateur, lié à gestion de la polyphonie), le "positionnement" du "sujet", locuteur ou énonciateur, la légitimation etlou la validité de ses dires, la fonction de "garant", le rôle fondationnel des interactions verbales où l'Un est toujours à construire en regard de l'Autre, l'argumentation dans la langue et l'argumentation rhétorique, mais l'ouvrage n'accorde que peu de place à la prise en charge des valeurs et des jugements de valeur. Sans doute est-ce l'une des formes que revêt la démarche parfaitement légitime qui conduit l'analyse du discours (désormais $\mathrm{AD}$ ) à se démarquer des théories de l'argumentation avec lesquelles elle partage néanmoins un commun intérêt pour les procédures de socialisation du langage en interaction.

Il ne s'agit pas ici de demander à l'AD d'être ce qu'elle ne souhaite pas être et donc d'intégrer l'angle d'attaque d'une éthique argumentative du discours dans ses axiomes fondationnels, mais de formuler l'hypothèse que les conditions de possibilité d'un questionnement éthique plus complexe existent d'ores et déjà dans les axiomes de l'AD, en dépit de quelques obstacles que je tenterai de répertorier et de définir.

Ma démarche sera donc la suivante: définir brièvement la conception de l'éthique qui sous-tend l'argumentaire de cet article, tenter de répondre aux questions suivantes: quelles sont les conditions de possibilité de l'intégration d'un questionnement éthique dans les axiomes de l'AD, mais aussi les obstacles qui s'y opposent? J'essaierai enfin d' augmenter la visibilité de ce que l'AD aurait à gagner si elle allait jusqu'au bout de ce qu'elle a d'ores et déjà pressenti: la problématisation de la difficile question de la "rectitude éthique" considérée par Plantin (2002, p. 72) comme l'une des "normes du discours argumentatif". La menace de la moralisation ${ }^{1}$ plane indéniablement sur ce genre de questionnement, mais la solution consiste-t-elle à le passer sous silence ou à le

Cf. la définition du "jugement" dans Charaudeau (1992, p. 587). L'auteur y précise que le locuteur " $s$ '(y) donne l'autorité morale de celui qui peut juger". L'ethos du juge 
marginaliser? Est-il impossible d'allier "souci éthique" et rationalité scientifique?

Ce questionnement aura le corpus suivant pour terrain d'investigation: une trentaine d'entrées du Dictionnaire d'analyse du discours, ${ }^{2}$ sélectionnées en fonction de points de convergence avec quelques concepts-clés de l'éthique ou de problématiques inhérentes à son champ, le numéro 117 de Langages, consacré aux "analyses du discours en France", l'ouvrage collectif Images de soi dans le discours, dirigé par Ruth Amossy, deux publications supplémentaires de Charaudeau: la Grammaire du sens et de l'expression (1992) et "Quand l'argumentation n'est que visée persuasive. L'exemple du discours politique", publié en 2005 dans Argumentation et communication, et Semen 22: "Énonciation et responsabilité dans les médias".

\section{BRÈVE DÉFINITION SÉLECTIVE DU CONCEPT D’ÉTHIQUE}

Le fait de commencer cet article par une brève présentation des traits distinctifs du concept d'éthique ne signifie pas que je souhaite parler d'un lieu extérieur au langage, lieu préétabli dans lequel je tenterais de le faire entrer de force...La raison d'être et la légitimité d'une approche éthique du discours est, me semble-t-il,

est souvent associé dans le discours du sens commun à l'image dévalorisante du donneur de leçons. Nous tenterons ici de justifier l'hypothèse qu'il existe une place, entre l'ethos de l'observateur impartial et celui du donneur de leçons, pour celui d'un sujet éthique qui argumente des jugements de valeur explicites ou implicites, conçus a priori comme réfutables.

2 Voici la liste des entrées consultées, classées par ordre alphabétique: "Actant", "Acte de langage", "Acteur", "Action”, "Action langagière", "Actions/ évènements", "Analyse du discours", "Argument", "Argumentation”, "Assertion”, "Auteur", "Autodésignation”, “Autorité”, "Captation”, “Crédibilité (stratégie de -)", "Discours”, "Émetteur”, "Énonciateur”, “Énonciation”, "Éthos”, “Identité”, "Idéologie”, “Incorporation”, "Individuation", "Légitimation", "Modalisation", "Locuteur", "Locuteur collectif", "Orientation argumentative", "Persuasion", "Point de vue", "Positionnement", "Rôle", "Subjectivité", "Sujet du discours", "Sujet parlant", "Valeur”. 
inscrite dans le fonctionnement même de la langue et du discours. Ce dernier ne sert pas uniquement à mettre en mots et donc en scène une vérité représentationnelle; il sert également à dire l'évaluation subjective affective etlou axiologique. Le fait que la catégorisation du "réel" puisse être orientée par des prises de position axiologiques et pas uniquement par des jugements de fait ou des assertions est considéré comme avéré dans la plupart des théories du langage actuelles quelle que soit la place accordée à la problématisation du rapport aux valeurs.

Les textes de référence dont s'inspire la brève définition annoncée ci-dessus sont les entrées "éthique" et "responsabilité" du dictionnaire des Notions philosophiques, publié sous la direction de Sylvain Auroux. ${ }^{3}$ Le concept de "sujet" y occupe une place centrale. Il y est certes présenté au prisme de l'opposition "individu। "milieu” (1990, p. 875), du contexte social dans lequel il évolue et des contraintes qui pèsent donc sur son autonomie (1990, p. 870, 2251-2), mais ceci ne fait pas de lui un être entièrement asservi à des injonctions doxiques. C'est la liberté, même partielle, du sujet, qui fait de lui un être autonome et moralement responsable (1990, p. 2250, 2253). Ce qui "fascine" ce sujet, ce ne sont pas uniquement les "évidences" rationalistes (1990, p. 875) consensuelles, mais la perspective de l'application de procédures d'évaluation rationnelles (ibid., p. 871) à un questionnement sur des valeurs. Ce dernier a la "rectitude éthique" pour objet, autrement dit le juste et l'injuste, le bien et le mal et non pas uniquement la véridiction. Rationalité et jugement de valeur subjectify sont considérés comme parfaitement compatibles.

Le sujet éthique a à se mesurer à des dilemmes qu'il doit résoudre en effectuant des choix, choix qui l'engagent (ibid., p. 870-

Les points communs et les différences entre les traits spécifiques du concept d'éthique, présentés ci-dessous, et les prises de position axiomatiques de l'AD seront commentés dans les développements de l'article où j'aborde les conditions de possibilité d'une éthique du discours, pressenties par l'AD, mais aussi la question des obstacles à l'intégration d'un tel questionnement. 
1,877 ) en tant qu'individu et qui doivent le mener à l'action. Si la morale est constituée par un système de principes transcendant, extérieur au sujet, qu'il saisit par la pensée et dont il est le récepteur en tant que membre d'une collectivité sociale, le questionnement éthique est inhérent au sujet, il "colle" "à chaque individualité" et correspond à une "exigence" intérieure de "fonder la conduite humaine, de donner un sens à la vie” (ibid., p. 875). L'éthique serait un mode de réflexion individuel sur l'agir humain ${ }^{4}$.

Le questionnement éthique est lié aux interactions verbales par des liens essentiels. La discussion contradictoire intersubjective (ibid., p. 871) y est considérée comme la condition préalable incontournable de la quête du juste, du vrai et du valide. L'éthique privilégie "l'action et, plus profondément, le langage."; l'orientation actuelle de la définition du concept serait liée au travaux de recherche de la philosophie analytique anglo-saxonne sur la dimension discursive des interactions (ibid., p. 875) par des liens essentiels.

Dernier point avant d'entrer dans le vif du sujet: l'importance accordée à la question de la responsabilité (ibid., p. 2252) et donc aux conséquences des systèmes de principes, aux modalités de leur concrétisation, à leur application à des cas concrets particuliers (p. $871,874,875)$.

\section{DE QUELQUES CONDITIONS DE POSSIBILITÉ D'UNE ÉTHIQUE DU DISCOURS, D'ORES ET DÉJÀ ÉTABLIES...}

Je me propose de présenter ici trois groupes de conditions de possibilité de l'intégration d'une éthique discursive dans le champ de l'AD. Le recours à la qualification de "condition de possibilité"

Ce point trouve un écho dans l'entrée "Actionslévénements (en narratologie)" du Dictionnaire d'analyse du discourse (2002, p. 26). La définition, rédigée par Jean-Michel Adam, s'y achève par cette conclusion: "Tout récit (...) peut être défini comme une interrogation portant sur les raisons d'agir, sur les degrés d'intentionnalité (motifs, buts) et donc sur la responsabilité des sujets". 
est dû, dans la plupart des cas, au fait que le mot "éthique" n'apparaît pas ou rarement sous la plume des auteurs du corpus analysé dans cet article et cela en dépit de recoupements ou de convergences entre des concepts etlou des positions axiomatiques similaires dans les deux champs.

Il s'agit, tout d'abord, de la place fondamentale accordée par l'analyse du discours à l'inscription discursive du sujet, à un questionnement sur son identité et aux stratégies de "prise en charge". Le second groupe a pour fin de rendre compte de développements épistémologiques récents qui ont trait à la problématisation de la responsabilité énonciative et de "la valeur argumentative" d' "un acte de discours", évaluée à l'aune de "la situation de communication dans laquelle se trouvent pris les partenaires d'un échange langagier" (Charaudeau, 2005, p. 29); le troisième concerne enfin un questionnement en gestation, qui a l'éthique professionnelle de l'analyste $\mathrm{du}$ discours pour objet.

\subsection{Mais qui est le "sujet"? Eléments pour un portrait éthique de l'énonciateur..}

Le concept de "sujet", qui joue un rôle éthique fondationnel, constitue le dernier des cinq points présentés par Dominique Maingueneau (2002, p. 202) ${ }^{5}$ comme caractéristiques des "tendances françaises en analyse du discours"; il y met l'accent sur "la réflexion" des "unités de la langue" et de leur "fonction discursive" "sur les modes

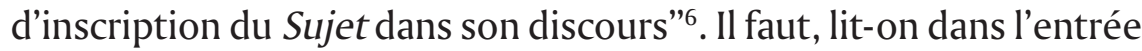
"Identité" (2002, p. 299), joindre "altérité" à "sujet", si l'on souhaite pouvoir "utiliser la notion d'identité en analyse du discours". "C'est à la mesure de la différence entre 'soi' et 'l'autre' que se constitue le sujet”(ibid.). Il y a donc, en l'occurrence, convergence entre un

5 J'emploierai désormais l'abréviation DictAD pour désigner cet ouvrage. Cf. également, l'entrée "Sujet du discours" (p. 554-5).

6 Ce sujet est l' "instance qui à la fois se pose comme source des repérages personnels, temporels, spatiaux et indique quelle attitude il adopte à l'égard de ce qu'il dit et de son 
axiome du questionnement éthique et de l'analyse du discours: le primat accordé à la co-construction du sens, à la confrontation verbale d'interlocuteurs ou même d'adversaires conçus et présentés comme inéluctablement solidaires. Le "positionnement"7 discursif de l'Un ne pourrait aboutir sans un affrontement dialogique réel ou imaginaire avec le point de vue de l'Autre. "Lidentité de positionnement, ajoute Charaudeau (ibid., p. 300), caractérise la position que le sujet occupe dans un champ discursif en rapport avec le système de valeurs qui y circulent, non pas de façon absolue, mais du fait des discours que lui-même produit. Ce type d'identité s'inscrit alors dans une formation discursive.". L'autonomie - et qui dit autonomie dit possibilité d'un questionnement éthique, obligations assumées librement - résiderait dans le fait que le sujet discursif puisse "mettre en œuvre" des "stratégies" dont il prend l'initiative et qui agencent les valeurs inhérentes aux discours qu'il "produit". Le sujet "énonciateur" est certes, pour l'analyse du discours, un "acteur social" (DictAD, 2002, p. 21-2) soumis à de multiples formes de contraintes, mais il reste néanmoins partiellement libre ${ }^{8}$, libre de ses choix discursifs, ${ }^{9}$ libre et donc responsable, en l'occurrence, de ses positionnements ou de ses "prises en charge" qui renvoient explicitement ou "en creux" à l' "univers de discours" "rejeté" (DictAD, 2002, p. 231, "Énonciateur").

Le sujet est le "garant" de la vérité qu'il met en scène; il construit, dans la trame de ses discours, l'ethos langagier qui devrait "légitimer son dire" (DictAD, 2002, p. 239). Chacun des traits

interlocuteur." "La réflexion sur les formes de subjectivité que suppose le discours est un des grands axes de l'analyse du discours." DictAD (2002, p. 189).

7 Cf. les définitions de ce terme dans les entrées "Individuation" (p. 308-9) et "Positionnement" (p. 453-4).

8 Cf. Charaudeau (2006, p. 30) et $\operatorname{DictAD}$ (2002, p. 340), "Légitimation", à propos de la notion d' "autorité personnelle".

9 Il y a ici convergence entre la conception du rapport choixlconstruction discursive et gestion de l'autonomie du sujet et l'hypothèse énoncée par le rhétoricien, E. Eggs, dans Images de soi dans le discours (1999, p. 33-4, 38-9, 41), hypothèse selon laquelle l'ethos "ne se montrerait qu'à travers les choix effectués" par le locuteur. 
spécifiques évoqués ci-dessus correspond à ceux du sujet éthique: identité constituée par des contraires solidaires: individualitél appartenance contraignante à un "milieu", autonomie $\backslash$ asservissement, liberté du choix et responsabilité individuelle. Le sujet du discours ou "actant" et le sujet de l'éthique ont tous deux enfin l'action pour enjeu, le premier est un "actant", "agent" et "instance" de "l'acte énoncif” (DictAD 2002, p. 16, "Actant”), le second, un acteur social dont la prise de position individuelle, médiatisée par le langage, est liée à des principes intériorisés qui gouvernent ses choix et les actes qui doivent en découler (Auroux, 1990, p. 870).

\subsection{De deux tournants épistémologiques: la prise en compte d'une responsabilité} énonciative, la conceptualisation de la valeur argumentative de l'acte de discours

La parution récente d'un numéro de la revue Semen (22, Novembre 2006) intitulé "Énonciation et responsabilité dans les médias", constitue un tournant dans la problématisation linguistique d'un concept d'éthique fondamental: la responsabilité. On peut voir dans les prises de position des coordinateurs, Alain Rabatel et Andrée Chauvin-Vileno et dans celles de l'ensemble des auteurs du volume une condition de possibilité de l'intégration d'un questionnement éthique dans le champ de l'analyse du discours. Cette intégration ne va pas de soi, comme le soulignent la plupart des auteurs: les concepts d'éthique et de responsabilité ne figurent pas dans les index de la majorité des ouvrages en sciences du langage, ils appartiennent à deux disciplines différentes: la philosophie et la linguistique. Les "observables" ou "traces", "marques" (Moirand, 2006, p. 48-9) linguistiques de la responsabilité énonciative en sont encore au stade du recensement, d'un recensement où figurent, entre autres, le choix des qualifications des acteurs et des actes ${ }^{10}$ ou le choix des modes de présentation du "discours des autres", le

10 Moirand (ibid., p. 45-6). Cf., également, Koren (ibid., p. 101-3 et 1996, p. 205-57), "Les dénominations du terroriste et de l'attentat". 
choix de lexèmes axiologiques ou l'axiologisation discursive de dénominations neutres, la concomitance ambigüe de dénominations qui criminalisent et qui légitiment, la mise en scène du discours sur les causes (Koren, 2006, p. 103-4), la "prise en charge" énonciative de la "construction d'une représentation discursive" et "la valeur illocutoire des actes", "inséparable de l'orientation argumentative des énoncés" (Adam et Lugrin, 2006, p. 127), la mise en scène polyphonique revisitée par Rabatel dont l'analyse aboutit à la conceptualisation de sept traits distinctifs de la responsabilité énonciative au nombre desquels on compte : "la responsabilité du choix de la dramatisation et des objets sur lesquels elle porte", "la responsabilité au plan de la sélection et de la combinaison des informations", "la responsabilité dans la gestion de la polyphonie", "la responsabilité du choix du cadre cognitif", "la responsabilité discursive, et non linguistique" (2006, p. 88-9).

Seconde condition de possibilité épistémologique: le fait que le DictAD comprenne une entrée "Argumentation" et la prise en compte, par exemple, par Charaudeau (2005, p. 34 et 38) de la nécessité et de la pertinence de l'intégration de la mise en scène argumentative dans la description d'actes de discours, spécifiques du discours politique. ${ }^{11}$ La définition du concept d'argumentation y confirme la pertinence de l'hypothèse selon laquelle le questionnement éthique serait inhérent à la rhétorique argumentative et de ce fait hors du champ des axiomes fondationnels de l'AD. Celle-ci considérerait l'analyse argumentative comme un prolongement logique et naturel de ses démarches interprétatives, mais entretiendrait simultanément avec elle un genre de rapports similaire au rôle attribué par le sujet du discours au discours de l'Autre: remplir la fonction d' "antigarant”. Ce qui retient en effet l'attention dans la définition de l'entrée "Argumentation", rédigée par Plantin (2002, p. 67-8), c'est

11 Les travaux de J-M. Adam et M. Bonhomme (1997), de J-M. Adam (1999), par exemple, l'ensemble des travaux de R. Amossy (dont Amossy, 1999 et 2006) et "La place de l'argumentation dans l'analyse du discours: Approches et enjeux contemporains" ici même, Plantin 2002a se situent au "carrefour" des deux disciplines. 
le fait que l'apparition du concept de "jugement" soit présentée comme un trait spécifique de la contribution de l'argumentation aux sciences du langage or qui dit "jugement", dit non seulement rapport aux faits, assertion, mais aussi rapport aux valeurs et prise de position qui engage la responsabilité de celui qui juge. ${ }^{12}$ Pour Perelman comme pour Eggs - nous le verrons ci-dessous dans " $\mathrm{La}$ primauté du métadiscours sur la mise en scène du 'Vrai"-les apparences d'"objectivité" et la prise de position subjective sont des composantes intégrales, concomitantes et indissociables, de toute prise de parole interactionnelle.

La prise en compte de la mise en scène argumentative et donc éthique des actes de discours est particulièrement perceptible, enfin, dans la contribution de Charaudeau (2005, p. 34, 38), au volume Argumentation et communication dans les médias. L'enjeu de persuasion qui caractérise le discours politique y est en effet présenté comme alliant souci de "véracité" et d' "influence" (p.34). Le sujet du discours politique ne se présenterait pas seulement comme le garant des imaginaires de vérité qu'il mobilise dans son argumentaire, mais ausi comme celui de l'appel aux valeurs qu'il cherche à faire partager; il serait à la fois responsable d'un parler vrai et d'un parler juste, même s'il n'existe pas de définition préalable absolue du vrai et du juste ni du "bon" et du "mauvais" argument. ${ }^{13}$ Imposer des réponses absolues serait le propre de la moralisation et pas du questionnement éthique.

12 Cf., entre autres, au sujet de la définition perelmanienne du jugement de fait, du jugement de valeur et du couple notionnel philosophique qu'ils forment, Ch. Perelman et L. Olbrechts-Tyteca (1970, p. 681-2). "La pratique et la théorie de l'argumentation sont, à nos yeux, affirment les auteurs du Traité, corrélatives d'un rationalisme critique, qui transcende la dualité jugements de réalité-jugements de valeur, et rend les uns comme les autres solidaires de la personnalité du savant ou du philosophe, responsable de ses décisions dans le domaine de la connaissance comme dans celui de l'action.".

13 Cf., au sujet de l'impossibilité de définir de façon irréfutable ce que serait un "bon" ou un "mauvais" argument l'analyse du point de vue de Perelman, qui nie la possibilité de définitions absolues en la matière, par Kerbrat-Orecchioni (1981, p. 43-4) et Charaudeau (2005, p. 34-5). 


\subsection{Problématisation de l'ethos du chercheur}

Les auteurs de "Discours en situation de travail”, Boutet, Gardin et Lacoste, paru en mars 1995 dans Langages 117,14 "Les analyses du discours en France", posent une question qui aurait pu constituer le point de départ d'un amendement de la doxa dominante dans le champ actuel des sciences du langage quant au devoir de nonintervention: ${ }^{15}$

"Le linguiste peut-il dès lors se comporter uniquement en 'linguiste': faire abstraction de cette intrication du langagier et du non-langagier et traiter les situations de travail comme un lieu de recueil de corpus au même titre qu'un square ou un arrêt d'autobus? Pour deux ensembles de raisons nous pensons que non. D'abord pour des raisons éthiques. Ce que les femmes et les hommes au travail engagent d'énergie, de pulsions, de souffrance rendrait mal venue une attitude de linguiste consistant à venir chercher là des corpus exotiques. Cependant, même si nous n'avions pas de semblables dispositions éthiques, les propriétés mêmes de l'empirie observée nous contraindraient à répondre négativement. (...) Les collectifs se construisent, se transforment ou se détruisent sur le terrain par les actions langagières des participants, ils ne sont pas seulement des êtres de papier préconstitués ou créés par un discours monologal. Nous trouvons des agents engagés collectivement dans des activités et obligés de se servir du langage pour se coordonner, co-construre un objet, un processus, réaliser une tâche.” (p.16)

14 Cf., à propos de la conception du rôle du chercheur, prônée par T. A. Van Dijk, "Idéologie", DictAD $\quad$ (p. 303). S. Bonnafous conclut la présentation historique du rapport de l'AD au concept en ces termes: "Ainsi (...) l'analyse du discours (la Critical Discourse Analysis en l'occurrence) doit participer activement, à la façon académique qui est la sienne, aux débats sociaux, et faire des recherches utiles à ceux qui en ont le plus besoin, plutôt qu'à ceux qui peuvent le plus payer" (Van Dijk, 1996, p. 27)".

15 Cf., à ce sujet, Auroux (1996, p. 327-8), Charaudeau (1992, p. 813) et Koren (2002, 2003, 2006, 2006a, p. 93-9). 
Ce qui traduit parfaitement ici le "souci éthique" (p.15) ${ }^{16}$ des trois chercheurs, c'est le fait de présenter leur position comme un choix et donc, "en creux", les positions contraires non pas comme des positions "rejetées", mais comme des options tout aussi valides. Ton péremptoire dogmatique et questionnement éthique seraient en fait incompatibles. Mais il y a davantage: "les propriétés mêmes de l'empirie observée”, argument qui incite à prendre en compte le milieu social observé, mais aussi l'ensemble de ses pratiques discursives. Les "dispositions éthiques" des chercheurs seraient donc ici l'un des résultats de l'observation de l' "empirie" et la condition de possibilité de descriptions qui choisissent d 'accorder une importance primordiale aux interactions verbales, en particulier, et à l'action, en général. On aurait pu, à la suite de ces trois auteurs, tenter d'appliquer cette vision de l'interprétation à tous les textes où sont formulés explicitement ou implicitement des jugements de valeur, nécessairement inscrits dans la trame discursive; mais il aurait fallu pour cela vouloir partager leurs "dispositions éthiques"...

Le réajustement de la question de la prise de position de l'analyste interprète, confronté au positionnement du discours médiatique est, plus récemment, l'une des préoccupations majeures de Semen 22. Le texte de présentation du numéro contient ainsi la déclaration suivante:

"Certes, le savant (ou le professeur) ne doivent pas transformer leur chaire en tribune: mais cela n'épuise pas le problème car les choix de tel ou tel paradigme scientifique, de méthodologie, de corpora ne font pas abstraction des convictions des savants. Aussi l'éthique de responsabilité ne fait-elle guère de sens que par rapport aux domaines de savoir bien balisés sur lesquels il y a consensus, mais perd de sa pertinence au-delà.” (p. 9, voir aussi ibid., p.12)

16 Cf., également, la suite de l'article (p. 17-20) et les références à l'éthique de l'analyste du discours dans les contributions de Beacco et Moirand, Branca- Rosoff et al (1995, p. 36, 64-5) à ce même numéro. 
Rabatel (2006, p. 77) y argumente la décision de compléter l'évocation des "routines professionnelles" et de l'application des "règles déontologiques" de l'écriture de presse par un examen critique de la "responsabilité des agents qui ont la responsabilité de faire évoluer les règles professionnelles ...ou de s'y conformer". Le rôle de l'analyste ne pourrait donc plus se limiter, dans le cas de certains discours comme les discours médiatiques ou politiques, à une "posture analytique"; il faudrait pouvoir poser sur les textes un "regard" éthique de scientifique "aux antipodes de la commode figure de l'imprécateur", regard qui pourrait impliquer, si nécessaire, "jugements de valeur", "attentes" et "insatisfactions" (ibid., p. 12). N'est-ce d'ailleurs pas le type de regard que pose Charaudeau (2006, p. 36) dans sa contribution au numéro, lorsqu'il recourt aux qualifications: "dérives énonciatives du discours journalistique", "dérives qui ne répondent plus à l'exigence d'éthique qui est celle de l'information citoyenne"?

\section{DE QUELQUES OBSTACLES MAJEURS À LA CONCRÉTISATION DES CONDITIONS DE POSSIBILITÉ}

Les conditions de possibilité réunies ci-dessus n'ont pas (ou pas encore.) abouti à l'institutionnalisation d'un questionnement éthique dans le champ des analyses du discours. Mon hypothèse est que cet état des lieux pourrait avoir au moins deux causes majeures: le rapport de l'AD à la vérité référentielle et aux valeurs.

\subsection{La primauté du métadiscours sur la mise en scène du "Vrai"}

Le rôle joué actuellement, dans la recherche en analyse du discours, par la problématisation de l'effacement énonciatif et des apparences de neutralité suffirait à prouver que les analystes du discours n'ont pas le "Vrai" pour objet, mais sa "mise en scène" 
inéluctablement marquée au sceau de la subjectivité. La prise en charge d'une vérité référentielle, la modalisation, ${ }^{17}$ par le sujet, de ses propres dires ou la prise en charge polyphonique (vs le rejet) de la vérité des dires des autres énonciateurs occupent cependant une place centrale dans les recherches actuelles. La question de la prise en charge de valeurs autres que le "Vrai" est marginalisée ou même passée sous silence. Il ne s'agit pas ici de nier l'importance de la question de la véracité qui est une valeur fondamentale ${ }^{18}$ sans laquelle il n'y aurait pas de relations sociales ni de crédibilité ${ }^{19}$ possibles. Mon hypothèse est donc la suivante: ce qui manque à cette conception dominante de l'éthique, c'est la reconnaissance explicite de la légitimité et de la nécessité d'un second type de questionnement concomitant et complémentaire qui aurait le parler juste et non le parler vrai pour objet..$^{20}$ L' entrée "Argumentation" du $\operatorname{Dict} A D$, rédigée par Plantin, souligne que la nécessité du choix d'une norme peut amener le sujet ou son interlocuteur à choisir entre "la véridiction" 21 et la "rectitude éthique" sans privilégier l'une au détriment de l'autre; et par "rectitude éthique", Plantin entend "conformité" dans une situation d'énonciation socio-historique, à

17 Cf., à propos de ce concept, l'entrée "Modalisation" du DictAD (2002, p. 382-3) et Kerbrat-Orechioni (1980, p. 109-10, 113-4) au sujet des verbes et des adverbes subjectifs modalisateurs qui référent à une évaluation en termes de "vrailfauxlincertain" et aux présupposés "incontestables" ou "irréfutables" qu'ils contribuent à énoncer.

18 Cf., quant à "la valeur de vérité", Perelman et Olbrechts-Tyteca (1970, p. 99-100).

19 Cf. "Crédibilité" dans DictAD (p. 154). Charaudeau y souligne que le concept est lié à un "jugement" "qui consiste à mesurer l'aptitude du sujet parlant à dire le vrai"; "la crédibilité est un fait de stratégie du discours qui, à l'instar des stratégies de légitimation" et de captation", consiste pour le sujet parlant à 'déterminer une position de vérité, de sorte qu'il puisse (...) être pris au sérieux"'.

20 L'assertion ou pratique discursive proche du jugement de fait pourrait, dans cette perspective, être considérée comme l'envers complémentaire de l'argument. C'est à l'aune du "Vrai" qu' elle est définie dans le DictAD (2002, p. 73). Cf., également, dans l'entrée "Argument" la distinction faite par Plantin (2002, p. 65) entre "Arguments vrais et vraisemblables".

21 Je vais tenter de démontrer ici que l'un des obstacles majeurs à l'intégration dans l'AD d'un questionnement éthique sur la prise en charge des valeurs est précisément le primat 
"un système de normes politico-morales". Perelman et OlbrechtsTyteca (1970, p. 103) présentent "la justice ou la véracité" comme des valeurs "abstraites" types, comme les deux pôles solidaires d'une même entité. ${ }^{22}$ Charaudeau (1992, p. 817), par contre, inclut la "justice" dans les valeurs spécifiques du "domaine de l'Ethique", mais il le dissocie de celui "de la Vérite" (ibid., p. 814). Or il serait impossible, affirme Plantin (2002, p. 72), de concevoir "une analyse du discours ordinaire" où l'argumentation ne serait pas "vécue par des sujets porteurs d'intérêts, de passions et de valeurs". L'une des raisons d'être fondationnelles des anciennes et des nouvelles rhétoriques n'est-elle pas la volonté de prouver que le logos ne nous sert pas uniquement à construire des procédures rationnelles vériconditionnelles, mais aussi à justifier et donc à rationaliser nos choix inéluctablement subjectifs, nos décisions axiologiques et même nos émotions ${ }^{23}$ ?

Le chapitre du rhétoricien Ekkehard Eggs, "Ethos aritotélicien, conviction et pragmatique moderne”, publié dans Images de soi dans

accordé à la prise en charge de la vérité référentielle ou de la vérité des dires du locuteur ou des énonciateurs intégrés dans la trame polyphonique de ses dires.

22 Cf. C. Kerbrat-Orecchioni (1980, p. 115), où l'auteur réfère au "jugement évaluatif" inhérent aux verbes subjectifs et associe, contrairement à la tendance dominante, les contraires solidaires de "l'axe" "bon|mauvais vs vrailfaux". La nouvelle rhétorique perelmanienne accorde certes une importance cruciale, dans le cadre des préliminaires de toute négociation argumentative, à l'établissement de faits et de vérités; il ne saurait y avoir argumentation sans accord préalable en la matière, mais elle insiste simultanément sur la nécessité de renforcer ces "objets" ("faits", "vérités” et "présomptions") qui requièrent un type d'accord d'ordre "universel”, par un recours à des "objets d'accord à propos desquels on ne prétend qu'à l'adhésion de groupes particuliers": "les valeurs", "les hiérarchies" et "les lieux du préférable" (p. 99). L'angle d'attaque du Traité permet donc de penser l'interaction et l'intrication de contraires solidaires.

23 Cf., à ce sujet, la mise en question du primat de la conception cartésienne de la raison et l'apologie d'une théorie de l'argumentation "qui admette l'usage de la raison pour diriger notre action et pour influer sur celle des autres" dans le Traité de l'argumentation (1970, p. 40, mais aussi l'argumentaire de Boudon (1995, p. 36-41) sur la rationalité des évaluations axiologiques qu'une doxa "positiviste" empêche de percevoir et de penser parce qu'elle 
le discours (1999, p. 31-59) me servira ici de référence quant à la nécessité de pouvoir penser la prise en charge du vrai sans pour autant passer sous silence celle du juste et de la dissociation entre le "bien" et le "mal". Eggs (1999, p. 43) y argumente en effet en faveur de l'interdépendance du vrai et du juste et de la nécessité de penser l' 'íntégrité discursive et rhétorique" comme une seule et unique entité. L'ethos langagier qui se construit au prisme de la véridiction et qui affiche une posture "neutre" et "objective" ne serait pas dissociable de son Autre adverse: "l'ethos moral" ou subjectif qui afficherait, quant à lui, le souci de paraître "équitable, sincère et solidaire" (ibid.). Eggs conclut:

Je suis (...) arrivé à une conclusion 'contradictoire' mais simple; on ne peut pas réaliser l'ethos moral sans réaliser en même temps l'ethos neutre, objectif ou stratégique. Il faut agir et argumenter stratégiquement pour pouvoir réaliser la sobriété morale du débat. Ces deux faces de l'ethos constituent donc deux éléments de la même procédure: convaincre par le discours." (ibid.). ${ }^{24}$

Il y aurait donc, dans le cas des interactions verbales, un prix à payer pour avoir droit à l'évaluation et au jugement: la pratique de la mise en scène du vrai, la référence au déjà-dit conventionnel ou stéréotypé, la révérence à une doxa qui donne toujours encore

donne le primat au Vrai et à la Raison. "Les systèmes de raisons" sous-tendent, affirme-til, et les jugements de valeur" et "les jugements de fait" (p. 37). Cf., enfin, dans le même ordre d'idée Tappolet, 2000.

24 Il existe ici une analogie entre la description des deux ethos contraires, mais solidaires et la conceptualisation de la question de l'hétérogénéité discursive dans les sciences du langage. Il y aurait, entre autres, au fondement du système du langage, un faisceau de tensions binaires contradictoires, mais inéluctablement intriquées, entre langue et discours, convention contraignante et autonomie, société et individu, stabilisation et déstabilisation, apparences d'objectivité et subjectivité affective ou évaluative, Un et NonUn, référenciation vériconditionnelle et acte de langage pragmatique ou évaluatif. Chacun des deux éléments infirmerait, relativiserait et légitimerait l'autre. Cf., au sujet de cette tension binaire, cette remarque ponctuelle, mais significative relevée dans le DictAD (p. 302): "Le terme d' 'idéologie' (...) s'accorde mal avec cette insistance nouvelle sur les phénomènes de contradiction et d'intrication". 
la primauté aux effets d'objectivité et à l'effacement énonciatif sur la prise de position explicite. ${ }^{25}$

Contrairement à la conception argumentative de l'intrication de deux modes de prise en charge contraires mais interdépendants, l'analyse du discours, comme on peut le voir dans les deux exemples types suivants, donne la primauté au positionnement vériconditionnel, et dans le cas des stratégies discursives et dans celui de la conceptualisation de l'argumentation. Charaudeau (1992, p. 814-5) classe "le domaine de l'Ethique", dans sa description des "procédés de la mise en argumentation", à la troisième place d'un recensement où figurent cinq "domaines d'évaluation"; "Le domaine de la Véritê" y occupe la première place. "L'Ethique" "définit" ce que "doivent être les comportements humains" "en termes de bien et de mal", le domaine de la "Vérité" est celui où se définirait "en termes de vrai et de faux" "ce qui concerne l'existence des êtres" et "ce qui relève $d u$ savoir comme principe unique d'explication des phénomènes du monde”. Les deux domaines y sont donc envisagés côte à côte, simultanément, au même titre que les domaines de l' "Esthétique", de l' "Hédonique" et du "Pragmatique". A chaque domaine d'évaluation, ses valeurs types, partagées par "les membres d'un groupe socio-culturel”. On trouve cependant, au début du développement qui a pour titre "La Mise en Argumentation", une dizaine de pages auparavant, dans "Le dispositif argumentatif", l'affirmation suivante: pour qu' un "processus argumentatif" se développe, il faut "que le sujet qui va argumenter prenne position par rapport à la véracité d'un Propos existant" (ibid., p. 803). "Il faut, poursuit Charaudeau, que le sujet dise pourquoi il est d'accord ou pas, ou, (...) qu'il apporte la preuve de la véracité de son Propos, qu'il développe ce que nous appelons un acte de Persuasion" (ibid., p. 804). C'est donc bien la "véracité" ou mise en scène de la vérité de ce qui "existe", qui constitue le point fort de la définition de la

25 cf. Koren (1997) au sujet de cette procédure de régulation de l'attribution du droit à la parole. 
"mise en argumentation". ${ }^{26}$ Il en est de même dans Charaudeau (2005, p. 29) où l'une des trois questions majeures qui "restent en débat", selon l'auteur, quant au champ de l'argumentation, "concerne le rapport entre raison et passion qui pose la question connexe du vrai et du faux". Que "raison" soit associé à "vrai” et "faux" n'a rien d'étonnant, mais "passion" aurait pu constituer simultanément le point de départ d'une autre "question connexe" suscitée par des liens avec le domaine du "bien", du "mal" ou du "juste" ou des commentaires sur la rationalisation des passions etlou la subjectivisation de la raison...

Seconde illustration: les dernières lignes d' "Ethos, scénographie, incorporation” où Maingueneau (1999, p. 100) conclut le chapitre en ces termes: "Lethos fait passer des schèmes qui sont censés agir à la marge des contenus mais qui imposent une figure à la source du Vrai: l'univers du discours prend corps dans la mise en scène d'un discours qui doit incarner sa vérité à travers son énonciation". L'analyste du discours y conceptualise la notion d'ethos "au-delà de la persuasion par des arguments"; il s'agit pour lui de "réfléchir sur le processus plus général de l'adhésion des sujets à une certaine disposition discursive" (ibid., p. 76). On pourrait en déduire "en creux" que lorsque l'on choisit de se situer hors du champ de l'argumentation (et je ne souhaite pas en disant cela rejoindre le camp de ceux qui considèrent le point de vue de Maingueneau sur le concept d'ethos comme une "déformation"ou même une "trahison" (ibid., p. 78), ce à quoi on renonce, consciemment

26 Cf., également, Charaudeau (1992, p. 784-5) où "argumenter" est défini comme "une activité discursive" envisagée "du point de vue du sujet", comme une "double quête": "quête de rationalité qui tend vers un idéal de vérité", "quête d'influence qui tend vers un idéal de persuasion". Cette seconde quête "se présente, affirme Charaudeau, comme faisant partie d'un processus rationnel et logique" (je souligne). Doit-on en déduire que la persuasion en est réduite à simuler le recours à des justifications rationalistes pour avoir le droit de dire la prise en charges de valeurs ? Ne peut-il y avoir de "visée rationalisante" de l'argumentation des valeurs ? Cf., enfin (ibid., p. 810), "Les 'positions' du sujet", où le primat est donné, dans la description de la prise de position argumentative, à la question de la "véracité". 
ou non, c'est à la problématisation de la prise en charge de valeurs autres que "le Vrai"27 et à la question de la "rectitude éthique".

\subsection{Du rapport de l'AD aux valeurs...}

Il ne s'agit pas ici de prétendre que l'analyse du discours ne problématise pas la question du rôle des valeurs dans les mises en mots discursives, mais d'examiner les tenants et aboutissants de son rapport aux valeurs. Maingueneau (1999, p. 79) affirme: "Caractère et corporalité du garant s'appuient" "sur un ensemble diffus de représentations sociales valorisées ou dévalorisées, de stéréotypes, sur lesquels l'énonciation s'appuie et qu'elle contribue en retour à conforter ou à transformer." "Conforter" et "transformer" prennent en compte la possibilité de deux ethos contraires, celui d'un sujet passif et soumis et celui d'un sujet actif autonome qui peut résister aux doxa et jouer un rôle énonciatif non-conformiste. Ce mode de présentation du rapport à la valorisation et à la dévalorisation n 'est cependant pas représentatif du discours le plus fréquent en la matière. ${ }^{28}$ Le sujet est présenté dans la majeure partie des cas comme condamné à "conforter" du déjà-valorisé ou "dévalorisé", tant est forte l'insistance du métadiscours des analystes sur les contraintes qui pèsent sur l'énonciateur, contraintes dues en premier lieu à la langue elle-même et aux valeurs qu'elles véhicule, ${ }^{29}$ aux genres discursifs, aux idées reçues et à la situation d'énonciation socio-historique. Le concept de "garant", "origine énonciative", "instance subjective", joue certes un rôle crucial dans les mises en scène discursives, mais son autonomie consisterait essentiellement

27 Cf. au sujet de la conceptualisation de la notion de "prise en charge" au prisme de la question de la responsabilité: A. Rabatel et A. Chauvin-Vileno (2006, p. 18-9).

28 Cf., à ce sujet, Rabatel et Chauvin-Vileno (2006, p. 16-9), "La notion de responsabilité en linguistique" et Koren (2006a, p. 97-9).

29 Il ne s'agit pas ici de prétendre, quant aux contraintes linguistiques, que le locuteur d'une langue est responsable du système qui la sous-tend et donc des lexèmes axiologiques qu'il comprend, même si "la langue, ce témoin juste de la complexité de nos sentiments 
dans le choix du genre de discours ou du type de scénographie mobilisé pour convaincre l'auditoire. Il ne s'agit pas ici de mettre la validité de ces analyses en question, mais de problématiser les conséquences d'une approche qui se veut complexe et nuancée, mais qui finit par marginaliser la question de l'autonomie du sujet, de la prise en charge des valeurs et donc l'option de la "transformation".

Quant à l'Autre qui joue un rôle fondamental et dans la définition philosophique du concept d'éthique et dans les axiomes de l'AD, il voit son rôle trop souvent réduit, via l'ethos ou "corps investi de valeurs historiquement spécifiées" (Maingueneau, 1999, p. 80), à celui d' "anti-garant", entendu comme "repoussoir" (ibid., p. 89). L'alternative positive existe certes sous les traits du "modèle valorisé" (ibid.), mais y a-t-il une place ici, en l'absence d'une conception argumentative de l'ethos, pour "l'interlocuteur" que Grize, cité par Plantin (2002, p. 67) dans l'entrée "Argumentation" du Dict $A D$, conçoit non comme "un objet à manipuler", mais comme "un alter ego auquel il s'agira de faire partager sa vision"? Consciemment ou non, l'analyse du discours favorise une conception polémique et non pas argumentative des interactions verbales où les locuteurs s'affrontent sur des questions liées à des systèmes de valeurs majoritairement préétablis. Le "rejet" semble l'emporter sur la négociation argumentée dans la définition du rôle joué par l' "interdiscours" dans la constitution de "l'univers de discours". On lit ainsi, à la fin de l'entrée "Énonciation” (p. 231), que celle-ci "revient à poser des frontières entre ce qui est 'selectionné" "et ce qui est rejeté. Ainsi se trouve dessiné en creux le champ de 'tout ce à quoi s'oppose ce que le sujet a dit"'. Autre justification de cette hypothèse,

et de nos dispositions éthiques, comme l'affirme Eggs (1999, p. 59), le (ce système d'évaluation de la responsabilité) donne à voir par l'existence de mots comme imprudent, insouciant, aventureux, risque-tout, bien ou mal intentionné, r'efléchi, sensé, judicieux...et pourquoi pas honnête, raisonnable, équitable.". Ce dont ce même locuteur est toutefois responsable, c'est du choix de la qualification retenue (Cf. à ce sujet, Rabatel et ChauvinVileno (2006, p. 17) et Rabatel (ibid., p. 89). 
les modalités des définitions de l'argumentation proposées par Charaudeau 1992. ${ }^{30}$ Lorsque le sujet choisit de "s'impliquer personnellement" dans un "questionnement" argumentatif, lit-on dans Charaudeau (1992, p. 812), "de le faire sien, de le défendre", ce questionnement se transforme en une "controverse dans laquelle les autres sujets qui argumentent et les autres arguments sont directement et explicitement mis en cause (voire mis en accusation) par des jugements de valeur plus ou moins passionnels, par des dénonciations, par des propos ironiques. On pourra parler dans ce cas d'argumentation polémique.". Celle-ci s'opposerait à l' "argumentation démonstrative", qui serait essentiellement à l'œuvre "dans les textes scientifiques ou didactiques". Le sujet ne s'y "implique pas personnellement" et n'y "met pas les autres personnellement en cause", si ce n'est par "le jeu des conclusions argumentatives". Une argumentation peut se "polémiser"; les traces discursives de cette transformation sont alors, effectivement, la dérive que constitue la substitution à un débat ad rem d'une attaque ad hominem, ${ }^{31}$ la violence verbale moralisatrice de l' "accusation", de la "dénonciation" ou de l'"ironie", mais elle cesse, le temps de cette polémisation, d'être elle-même. Le proposant, l'opposant et le tiers peuvent s'affronter violemment, mais à propos de l'objet du dissensus et sans perdre de vue leur intérêt commun: aboutir à un consensus, même partiel. Ce qui manque ici, c'est une troisième option qui occuperait l'espace laissé vide entre "engagement polémique" et "non engagement" de l' argumentation "démonstrative" "raisonnante": l'option proposée, par exemple, par "la nouvelle rhétorique" 32 perelmanienne ou celle défendue par Raymond Boudon (1995), option d'une prise de position éthique subjective justifiée par des jugements de valeur

30 Cf., également, Charaudeau (2002, p. 93-4) "II. Captation vs Subversion" et ibid. (p. 429), où Charaudeau présente le recours à l'option de la polémique comme l'alternative à laquelle recourrait le proposant de l'argumentation en cas d'échec.

31 Cf., au sujet de cette distinction entre "l'argumentation sur l'objet" et 'l'argumentation de soi”, Plantin (2003, p. 406-7).

32 Cf.Perelman et Olbrechts-Tyteca (1970, p. 17-9) "Démonstration et argumentation". 
qui peuvent être passionnés, mais sans renoncer pour autant à toute rationalité. Le sujet perelmanien est un être de discours "engagé", ${ }^{33}$ qui se doit de trancher au terme d'interactions verbales qui impliquent a priori de considérer l'Autre et comme un opposant et comme un "alter ego". Là où le discours polémique voit une personne à "incorporer" ou à exclure, le discours argumentatif voit un opposant à persuader, qui occupe une des places d'un jeu de rôles où les fonctions de proposant et d'opposant sont interchangeables.

Charaudeau (2002, p. 429-30) affirme à la fin de l'entrée "Persuasion" définie au prisme de "L'analyse du discours": "L'analyse de la persuasion appelle celle de la conversion, des discours de convertisseurs et de convertis qui en marquent un aboutissement". Plantin (2003, p. 407) achève sa contribution au volume collectif La parole polémique par cette remarque qui pourrait contribuer à la justification de mon hypothèse du primat d'une conception polémique des interactions verbales en analyse du discours: "Il conviendrait également de mettre en chantier de nouvelles réflexions sur le thème persuader, convaincre et convertir, car l'argumentation polémique cherche moins à persuader ou à convaincre qu'à convertir". "Convertir" n'est pas pour Plantin dans le prolongement d' "argumenter"; ${ }^{34}$ il existe, à ses yeux, entre ces deux notions des différences considérables.

Les interactions verbales peuvent aboutir à un échec, mais elles peuvent aussi faire naître un sentiment d' "euphorie". Maingueneau (1999, p. 100) conclut ainsi le chapitre "Ethos, scénogra-

Cf. ibid. (1970, p. 78-83), "Argumentation et engagement".

34 Plantin (2002, p. 599) reconnaît qu'il existe entre la notion de valeur et l'orientation polémique d'un discours des liens fort proches attestés par la structure même du lexique où les mots" exprimant des valeurs" constituent "un gigantesque réservoir de couples polémiques" "antonymiques" comme "savoir\ignorance", véritélmensonge", libertélopression"..etc..Lorientation argumentative inhérente à ces mots n'est cependant pas condamnée automatiquement à aboutir à la polémisation des discours. Les concepts de valeur et de polémique sont parfaitement dissociables; leur dissociation dépend des choix éthiques du sujet du discours. 
phie, incorporation": "Par sa manière même de s'énoncer le discours montre une régulation euphorique du sujet qui le soutient et du lecteur qu'il prétend avoir." Cette euphorie serait celle d'un sujet qui aurait pour visée d' "incorporer" un lecteur invité par le ton ${ }^{35}$ et le mouvement du texte à fusionner et à s'identifier avec lui. L' actant d'interactions argumentatives a aussi "des affects" que Plantin (2003, p.386) décrit en ces termes: "allégresse du Proposant ('J’ai une idée!'), indignation de l'Opposant ('On touche à mes valeurs!), perplexité du Tiers ("On ne sait plus où on en est?)". Le sujet du discours oscille entre "rejet" et incorporation, le proposant, l'opposant et le tiers négocient et co-construisent des accords consensuels. Ce que l'on risque de perdre, entre autres, en dissociant ces deux visions des interactions langagières et en donnant la primauté à l'ordre du polémique, ${ }^{36}$ c'est ce que Ruth Amossy (1999, p. 152-4) souligne dans "Au carrefour des disciplines": "un déplacement important de la notion d'efficacité discursive" et la prise en compte d'un type d'auditoire avec lequel on accepte de construire et de partager un "espace argumentatif" où se négocient des vérités, mais aussi des valeurs rationalisées par des justifications, à l'abri, le temps d'une négociation authentique, de l'ordre de la "domination" ou de la "captation" par "subversion". ${ }^{37}$

35 Cf., quant à l'articulation des concepts de ton, d'ethos et de "positionnement discursif”, Maingueneau (1999, p. 80). Cf., également, le rapprochement proposé par Moirand (2006, p. 47-9, et 51) entre "intonation" et la notion d'“éclairage", "empruntée à la conception de l'argumentation de Jean-Blaise Grize".

36 Donner la primauté à la prise en charge du Vrai conduirait presque naturellement à donner la vedette à une conception rationaliste du sujet, prêt etlou décidé à exclure tout adversaire qui refuserait de reconnaître qu'il détient le monopole de la définition d'une vérité historique absolue.

37 Cf. l'entrée "Captation” du DictAD (2002, p. 93-4). 


\section{EN GUISE DE CONCLUSION...}

La conceptualisation d'une éthique du discours et son intégration dans le champ de l'AD pourraient contribuer à accomplir la visée évoquée à la fin de l"'Avant-propos" du DictAD (2002, p. 12): choisir pour "objet d'étude" la "totalité des productions verbales, dans leur multiplicité", préparer la réflexion sur le fait que l'homme n'est pas seulement un "être de langage", mais aussi "un homme de discours". Si tel est le corpus que l'AD voudrait avoir pour objet et si "l'étude même du discours" tend à devenir "une dimension à part entière de l'activité sociale" (Langages, 117, p. 11), il est plus que jamais nécessaire de pouvoir penser et éventuellement évaluer les stratégies discursives auxquelles les locuteurs recourent pour juger et justifier leurs jugements de valeur et les effets éventuels de ces stratégies. Le sujet du discours pratique l'effacement énonciatif de préférence à la prise de position explicite et multiplie les effets d'objectivité conformément aux injonctions de la doxa, mais cela ne modifie pas le fait, comme l'affirme Kerbrat (1980, p. 82), ${ }^{38}$ que: "la plupart des énoncés produits en langue naturelle se caractérisent par la présence plus ou moins massive des axiologiques, et les comportements langagiers, par le souci constant de dresser entre le bien et le mal une barrière terminologique", "besoin d'encodage qui se répercute au décodage sous la forme de ce réflexe interprétatif dont on fait constamment l'expérience: que pense celui qui parle de l'objet dont il parle? est-il 'pour', est-il 'contre'?", “il arrive bien souvent que l'arsenal argumentatif déployé par tel ou tel discours n'ait pas tant pour but de mettre au jour une 'vérité' quelconque que de couvrir et légitimer a posteriori des parti-pris indéracinables". Les "diabolisations" et les "angélisations" qui envahissent les espaces discursifs médiatiques actuels et leurs éventuelles conséquences

38 Cf., également, l'entrée "Valeur" du DictAD (2002, p. 599), où Plantin affirme: "L'aspiration à un langage 'non biaisé', c'est-à-dire à l'élimination complète des jugements de valeur (subjectifs, émotionnels, orientés) au profit des seuls jugements de fait, ne pourrait être satisfaite qu'en renonçant au langage naturel pour une langue formelle ou angélique.". 
suffiraient à elles seules à justifier l'intégration, aux côtés d'une analyse descriptive de la "mise en scène" idéologique du "Vrai", d'un questionnement sur la "rectitude éthique".

On voit apparaître dernièrement dans la trame de travaux de recherches en analyse du discours, au-dessus de tout soupçon, des qualifications comme "dérive" (Charaudeau, 2006, p. 36), "manipulation" (Adam, 1999, p. 102-3) ou "responsabilité" (Rabatel Chauvin-Vileno et l'ensemble des auteurs de Semen 22). La présence de ces termes subjectifs axiologiques dans la trame de travaux scientifiques est un symptôme que l'analyse du discours devrait problématiser et conceptualiser. Le "souci éthique" est à l'étroit dans une conception déontologique rigide; le temps n'est-il pas venu de reconnaître que la prise en charge du vrai et du juste ont besoin l'une de l'autre pour se légitimer réciproquement? L'émergence des concepts de "dérive", "manipulation" ou "responsabilité" témoigne du fait que la notion de "visée" de l'acte d'énonciation, empruntée par l'analyse du discours aux théories pragmatiques du langage, peut conduire, par exemple, à aller plus loin dans la voie d'un questionnement éthique et à demander au sujet du discours d'être comptable de ses prises de position quant au "vrai", mais aussi quant au "juste", au "bien" et au "mal". On connaît la mise en garde de bien des déclarations axiomatiques: le linguiste, analyste du discours ou de la mise en mots argumentative, ne serait pas armé scientifiquement pour se prononcer sur les effets perlocutoires des actes de langage, argument puissant et redoutable du fait de sa rigueur et de la place légitime qu'il accorde à la question primordiale de la délimitation de l'objet d'une discipline. Mais en quoi l'interprétation évaluative du positionnement d'un texte idéologique, par exemple, et la problématisation de ses enjeux et de ses éventuels effets perlocutoires, dûment justifiée par une analyse linguistique, discursive et argumentative explicite, pourraient-ils constituer une trangression épistémologique?

S'il n'existe ni dans le domaine de l'éthique ni dans celui de l'argumentation rhétorique de définition absolue du "juste", du 
"bien”, du "mal” et du vrai ou du fait, cela n'implique pas qu'il faille marginaliser le questionnement éthique, y renoncer ou pratiquer un silence métalinguistique rationaliste rigide sur tout ce qui concerne l'évaluation des valeurs, mais que le sujet du discours est à la fois un sujet ancré dans une situation socio-historique concrète et contraignante et un sujet autonome, libre de choisir la mise en scène du vrai etlou des jugements de valeur dont il aura à assumer la responsabilité.

\section{BIBLIOGRAPHIE}

ADAM, J-M.; BONHOMME, M. (1997) L'argumentation publicitaire. Paris: Nathan.

ADAM, J-M. (1999) Images de soi et schématisation de l'orateur; Pétain et De Gaulle en juin 1940. In: AMOSSY, R. (éd.). Images de soi dans le discours. Lausanne/Paris: Delachaux et Niestlé.

ADAM, J-M.; LUGRIN, G. (2006) Effacement énonciatif et diffraction co-textuelle de la prise en charge des énoncés dans les hyperstructures journalistiques. In: RABATEL, A.; CHAUVIN-VILENO, A. (éds.). Semen, 22, p. 127-44.

AMOSSY, R. (2006) L'argumentation dans le discours. 2è éd. Paris: Armand Colin.

. (1999) L'ethos au carrefour des disciplines; rhétorique, pragmatique, sociologie des champs. In: R. AMOSSY (éd.). Images de soi dans le discours. Lausanne/Paris: Delachaux et Niestlé.

AUROUX, S. (1996) La philosophie du langage. Paris: Presses Universitaires de France. . (éd.). (1990) Les notions philosophiques, t.1, p. 1-1517 et t. 2, p. 1519-3297. In: $\overline{\mathrm{JACOB}}$, A. (éd.). Encyclopédie Philosophique Universelle.

BEACCO, J-C.; MOIRAND, S. (1995) Autour des discours de transmission de connaissances. Langages, 117, Les analyses du discours en France, p. 32-53.

BOUDON, R. (1995) Sens et raisons: théorie de l'argumentation et Sciences Humaines. Hermès, 16, Argumentation et Rhétorique, p. 29-43.

BOUTET, J.; GARDIN, B.; LACOSTE, M. (1995) Discours en situation de travail. Langages, 117, Les analyses du discours en France, p. 12-31.

BRANCA-ROSOFF; S. COLLINOT, A.; GUILHAUMOU, J.; MAZIÈRE, F. (1995) Questions d'histoire et de sens. Langages, 117, Les analyses du discours en France, p. 54-66.

CHARAUDEAU, P. (2005) Quand l'argumentation n'est que visée persuasive. L'exemple du discours politique. In: M. BURGER; G. MARTEL (éds.). Argumentation et communication dans les médias. Québec: Éditions Nota Bene.

. (1992) Grammaire du sens et de l'expression. Paris: Hachette.

CHARAUDEAU, P.; MAINGUENEAU, D. (2002) Dictionnaire d'analyse du discours. Paris: Seuil. 
Filol. lingüíst. port., n. 9, p. 251-278, 2007.

EGGS, E. (1999) Ethos aristotélicien, conviction et pragmatique moderne. In: AMOSSY, R. (éd.). Images de soi dans le discours. Lausanne-Paris: Delachaux et Niestlé.

KERBRAT-ORECHIONNI, C. (1981) Argumentation et mauvaise foi. In: L'argumentation. Lyon: PUL, p. 41-63.

. (1980) L'énonciation: de la subjectivité dans le langage. Paris: Armand Colin.

KOREN, R. (2006) Quels risques pour quelles prises de position 'normatives'? Questions de communication, 9, p. 195-205.

. (2006a) La responsabilité des Uns dans le regard des Autres: l'effacement énonciatif au prisme de la prise de position argumentative. Semen, 22, p. 87-94.

. (2003) L'engagement de l'Un dans le regard de l'Autre: le point de vue d'une linguiste. Questions de communication, 4, p. 271-7.

. (2002) 'La nouvelle rhétorique': 'technique' et/ou 'éthique du discours; le cas de l'engagement du chercheur. In: KOREN, R.; AMOSSY, R. (éds.). Après Perelman: quelles politiques pour les nouvelles rhétoriques?L'argumentation dans les sciences du langage. Paris: L'Harmattan, p. 197-228.

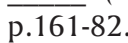

(1997) Remarques à propos d'un groupe nominal: le miroir virulent. Scolia, 10,

. (1996) Les enjeux éthiques de l'écriture de presse et la mise en mots du terrorisme. Collection Sémantiques. Paris: L'Harmattan.

LANGAGES 117, Mars 1995, "Les analyses du discours en France", sous la direction de Dominique Maingueneau.

MAINGUENAU, D. (1999) Ethos, scénographie, incorporation. In: AMOSSY, R. (éd.). Images de soi dans le discours. Lausanne/Paris: Delachaux et Niestlé.

. (1995) Présentation. Langages, 117, p. 5-11.

MOIRAND, S. (2006) Responsabilité et énonciation dans la presse quotidienne; questionnements sur les observables et les catégories d'analyse. Semen, 22, p. 45-59.

PERELMAN, C.; OLBRECHTS-TYTECA, L. (1970) Le traité de l'argumentation. 4è éd. Bruxelles: Éditions de l'Université de Bruxelles.

PLANTIN, C. (2003) Des polémistes aux polémiqueurs. In: DECLERCQ, G.; MURAT, M.; DANGEL, J. (éds.). La parole polémique. Paris: Honoré Champion, p. 377-408.

. (2002) "Argumentation", "Valeur”. In: CHARAUDEAU, P.; MAINGUENEAU, D. (éds.). Dictionnaire de l'analyse du discours. Paris: Seuil.

. (2002a) Analyse et critique du discours argumentatif. In: KOREN, R.; AMOSSY, R. (éds.). Après Perelman; Quelles politiques pour les nouvelles rhétoriques? Largumentation dans les sciences du langage. Paris: L'Harmattan.

RABATEL, A.; CHAUVIN-VILENO, A. (2006) La question de la responsabilité dans l'écriture de presse. Semen, 22, p. 7-27.

RABATEL, A. (2006) L'effacement de la figure de l'auteur dans la construction événementielle d'un 'journal' de campagne électorale et la question de la responsabilité en l'absence de récit primaire. Sêmen, 22, p. 77-92. 
KOREN, Roselyne. L'analyse du discours à l'aune d'un questionnement éthique

. (2006) Prise en charge et imputabilité, ou la prise en charge à responsabilité limitée..., à paraître. Semen, 22, Novembre 2006, "Énonciation et responsabilité dans les médias", coordonné par Alain Rabatel \& Andrée Chauvin-Vileno.

TAPPOLET, C. (2000) Emotions et valeurs. Paris: Presses Universitaires de France.

RESUMO: O Dictionnaire de l'analyse du discours, organizado por Charaudeau \& Maingueneau (2002), apesar de definir, por exemplo, conceitos como "sujeito", "identidade", "ethos", "argumentação", "valor", "posicionamento", "responsabilidade” pela "verdade” referencial ou por aquela dos dizeres dos outros enunciadores integrados na trama polifônica do discurso etc., não contém a entrada "Ética". Tudo acontece como se a AD tivesse pressentido e instaurado as condições de possibilidade de um questionamento ético sem, no entanto, desejar que esse questionamento conseguisse integrar, em seu campo, a "responsabilidade" pela "verdade", a "responsabilidade" pelos julgamentos de valor por parte do "locutor" ou dos outros "sujeitos do discurso". Este artigo tem como finalidade problematizar esses paradoxos e tentar mostrar que eles estão ligados a uma das maiores diferenças que separam a AD do campo da argumentação: a relação com o "Verdadeiro", com os valores e interações linguageiras conflituosas.

PALAVRAS-CHAVE: Análise do discurso; argumentação; ética; racionalidade; o Outro na AD e na textualização argumentativa; ética do pesquisador das Ciências da Linguagem. 